\title{
Plane Partition Polynomial Asymptotics
}

\author{
Robert P. Boyer and Daniel T. Parry
}

\begin{abstract}
The plane partition polynomial $Q_{n}(x)$ is the polynomial of degree $n$ whose coefficients count the number of plane partitions of $n$ indexed by their trace. Extending classical work of E.M. Wright, we develop the asymptotics of these polynomials inside the unit disk using the circle method.
\end{abstract}

\section{Introduction}

A plane partition $\pi$ of a positive integer $n$ is an array $\left[\pi_{i, j}\right]$ of nonnegative integers such that $\sum \pi_{i, j}=n$ while its trace is the sum of the diagonal entries $\sum \pi_{i, i}$. The asymptotics of the plane partition numbers $P L(n)$, the number of all plane partitions of $n$, was found by Wright [8] in 1931 using the circle and saddle point methods. In this paper, we study the asymptotics of polynomial versions $Q_{n}(x)$ of the plane partitions of $n$ given by

Definition 1. Let $Q_{n}(x)$ be the $n$-th degree polynomial given by $\sum_{k=1}^{n} p p_{k}(n) x^{k}$ where $p_{k}(n)$ is the number of plane partitions of $n$ with trace $k$. These polynomials have generating function

$$
P(x, u)=\prod_{m=1}^{\infty} \frac{1}{\left(1-x u^{m}\right)^{m}}=1+\sum_{n=1}^{\infty} Q_{n}(x) u^{n}
$$

(see [1, 6]).

In adapting the circle method to develop the asymptotics of these polynomials (see Section 4), we needed to introduce the sequence $\left\{L_{k}(x)\right\}$ of functions to describe the dominant contributions to their asymptotics where

$$
L_{k}(x)=\frac{1}{k} \sqrt[3]{2 L i_{3}\left(x^{k}\right)}
$$

and $L_{i}(x)$ is the trilogarithm function given by $\sum_{n=1}^{\infty} x^{n} / n^{3}$.

An important first step in the asymptotic analysis of the polynomials was obtained in our paper [3] where we determined exactly when $\Re L_{m}(x)(m=1,2)$ dominates $\Re L_{k}(x)$, for $k \neq m$, inside the unit disk (see [3] ).

1991 Mathematics Subject Classification. Primary: 11C08 Secondary: 11M35, 30C55, 30E15

Key words and phrases. Plane partition, polynomials, asymptotics, circle method, trilogarithm, phase. 
A primary motivation for us to develop the asymptotics was to find the limiting behavior of the zeros of the plane partition polynomials which is described in detail in our paper [4].

\section{Factorization of the Generating Function $P(x, u)$}

We are interested in the behavior of $P(x, u)$ in a neighborhood of $u=e^{2 \pi i h / k}$ inside the unit disk $\mathbb{D}$, where $h$ and $k$ are relatively prime. To start we expand the logarithm of the generating function $\ln P(w, u)$

$$
\ln P(w, u)=\sum_{\ell=1}^{\infty} \frac{x^{\ell}}{\ell} \frac{u^{\ell}}{\left(1-u^{\ell}\right)^{2}}
$$

for $x, u \in \mathbb{D}$. Next with $u=e^{-w+2 \pi i h / k}$, with $\Re(w)>0$, we introduce two functions

$$
A_{h, k}(x, w)=\sum_{k \nmid \ell} \frac{x^{\ell}}{\ell} \frac{e^{-\ell w+2 \pi i \ell h / k}}{\left(1-e^{-\ell w+2 \pi i \ell h / k}\right)^{2}}, \quad B_{h, k}(x, w)=\sum_{\ell=1}^{\infty} \frac{x^{k \ell}}{k \ell} \frac{e^{-\ell k w}}{\left(1-e^{-\ell k w}\right)^{2}} .
$$

Then the generating function decomposes as

$$
\ln \left[P\left(x, e^{-w+2 \pi i h / k}\right)\right]=A_{h, k}(x, x, w)+B_{h, k}(x, w) .
$$

Note that when $k=1, A_{h, k}(x, w)=0$ and that

$$
A_{h, k}(x, 0)=-\frac{1}{4} \sum_{k \nmid \ell} \frac{x^{\ell}}{\ell} \csc ^{2}(\pi \ell h / k), \quad k \geq 2 .
$$

We need three additional functions $\Psi_{h, k}(x, w), \omega_{h, k, n}(x)$, and $g_{h, k}(x, w)$ where

$$
\ln \left[\omega_{h, k, n}(x)\right]= \begin{cases}\frac{1}{12 k} \ln \left(1-x^{k}\right)-A_{h, k}(x, 0)-2 \pi i n h / k, & k \geq 2 \\ \frac{1}{12} \ln (1-x), & k=1\end{cases}
$$

$$
g_{h, k}(x, w)= \begin{cases}{\left[A_{h, k}(x, w)-A_{h, k}(x, 0)\right]+\left[B_{h, k}(x, w)-\Psi_{h, k}(x, w)-\frac{1}{12 k} \ln \left(1-x^{k}\right)\right],} & k \geq 2 \\ B_{0,1}(x, w)-\Psi_{0,1}(x, w)-\frac{1}{12} \ln (1-x), & k=1\end{cases}
$$

By construction, $P\left(x, e^{-w+2 \pi i h / k}\right)$ admits a factorization as follows.

Proposition 2. Let $h, k, n$ be nonnegative integers such that $(h, k)=1$. If $\Re w>0$ and $x \in \mathbb{D}$, then the generating function $P\left(x, e^{-w+2 \pi i h / k}\right)$ factors as

$$
P\left(x, e^{-w+2 \pi i h / k}\right)=\omega_{h, k, n}(x) e^{2 \pi i n h / k} e^{\Psi_{h, k}(x, w)} e^{g_{h, k}(x, w)},
$$

where $\omega_{h, k, n}(x), \Psi_{h, k}(x, w)$ and $g_{h, k}(x, w)$ are given in equations (4), (5), (6).

The key in using this factorization for the circle method is the following bound. 
Proposition 3. Assume that $0<|x|<1$ and $\Re w>0$. (a) If $\Im w \neq 0$ as well, then there exists $M>0$ such that

$$
\begin{aligned}
& \left|g_{h, k}(x, w)\right| \leq \frac{2|w|}{1-|x|}\left[k^{3}+\frac{|x|^{\frac{\pi}{|\Im| \Im w}}}{1-e^{-\frac{\pi \Re w}{k \mid \Im w}}}\right]
\end{aligned}
$$

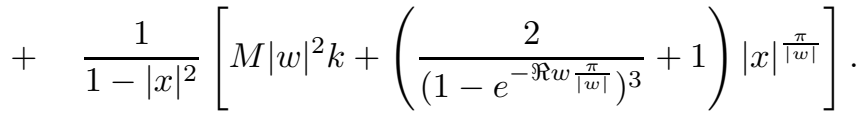

(b) If $w$ is real and positive, then there exists $M>0$ such that

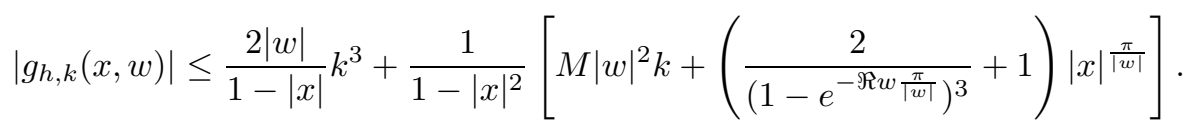

The proof is given in the following two lemmas.

Lemma 4. Let $k \geq 2$ and $\Re w>0$. (a) If $\Im w \neq 0$, then

$$
\left|A_{h, k}(x, w)-A_{h, k}(x, 0)\right| \leq \frac{2|w|}{1-|x|}\left[k^{3}+\frac{|x|^{\frac{\pi}{k|\Im w|}}}{1-e^{-\frac{\pi \Re w}{k|\Im w|}}}\right]
$$

(b) When $\Im w=0,\left|A_{h, k}(x, w)-A_{h, k}(x, 0)\right| \leq \frac{2|w|}{1-|x|} k^{3}$.

Proof. (a) For fixed $w$ and $x$, consider the function of $t, A_{h, k}(x, w t)$. By the Mean Value Theorem, we find

$$
\begin{aligned}
\left|A_{h, k}(x, w)-A_{h, k}(x, 0)\right| & \leq \sup _{0<t<1}\left|\frac{d}{d t} A_{h, k}(x, w t)\right| \\
& \leq|w|\left|\sum_{k \nmid \ell} x^{\ell} \frac{e^{-\ell w t+2 \pi i \ell h / k}\left(1+e^{-\ell w t+2 \pi i \ell h / k}\right)}{\left(1-e^{-\ell w t+2 \pi i \ell h / k}\right)^{3}}\right| \\
& \leq 2|w| \sum_{k \nmid \ell} \frac{|x|^{\ell}}{\left|e^{-w t \ell+2 \pi i \ell h / k}-1\right|^{3}} .
\end{aligned}
$$

For $0<t<1$ and $\ell \nmid k$ such that $\ell|\Im w| t<\pi / k$, we have the bound

$$
\frac{1}{\left|e^{-w t \ell+2 \pi i \ell h / k}-1\right|} \leq \frac{1}{|\sin (2 \pi \ell h / k-\Im w t \ell)|} \leq|\csc (\pi / k)| \leq k / 2
$$

since $|\sin (y)| \leq\left|e^{x+i y}-1\right|$. On the other hand, if $\ell \geq \frac{\pi}{k|\Im w| t}$, we see that

$$
\frac{1}{\left|e^{-w t \ell t+2 \pi i \ell h / k}-1\right|} \leq \frac{1}{1-e^{-\Re w t \ell}} \leq \frac{1}{1-e^{-\frac{\pi \Re w}{k|\Im w|}}}
$$


Combining these last two bounds, we can complete the proof:

$$
\begin{aligned}
\left|\frac{d}{d t} A_{h, k}(x, w t)\right| & \leq 2 \sum_{k \nmid \ell} \frac{|x|^{\ell}}{\left|e^{-w t \ell+2 \pi i \ell h / k}-1\right|^{3}} \\
& \leq \sum_{\substack{k \nmid \ell \\
\ell<\pi /[k|\Im w| t]}} 2 k^{3}|x|^{\ell}+\frac{2}{\left(1-e^{\left.-\frac{\pi \Re w}{k|\Im w|}\right)^{3}}\right.} \sum_{\substack{k \nmid \ell \\
\ell \geq \pi /[k|\Im w| t]}}|x|^{\ell} \\
& \leq 2 k^{3} \frac{1}{1-|x|}+\frac{2}{\left(1-e^{-\frac{\pi \Re w}{k \mid \Im w} \mid}\right)^{3}} \frac{|x|^{\frac{\pi}{k|\Im w| t}}}{1-|x|} \\
& \leq \frac{2}{1-|x|}\left[k^{3}+\frac{|x|^{\frac{\pi}{\mid \Im} w \mid}}{1-e^{-\frac{\pi \Re w}{k|\Im w|}}}\right] .
\end{aligned}
$$

(b) Both $|x|^{\frac{\pi}{|\Im w|}}$ and $e^{-\pi \frac{\Re w}{k|\Im w|}}$ go to 0 as $\Im w \rightarrow 0$, so the bound in part (b) follows from part (a).

Lemma 5. Assume that $0<|x|<1$ and $\Re w>0$. There exists $M>0$ such that

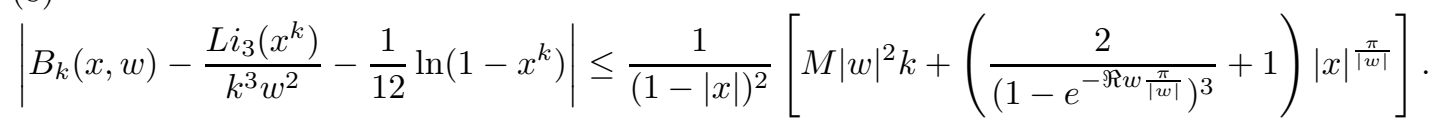

Proof. We begin by expanding the left-hand side of (8) as a series

$$
\sum_{\ell=1}^{\infty} \frac{x^{k \ell}}{k \ell}\left[\frac{e^{-w k \ell}}{\left(e^{-w k \ell}-1\right)^{2}}-\frac{1}{\ell^{2} k^{2} w^{2}}+\frac{1}{12}\right] .
$$

For $|k w \ell|<\pi$, there exists $M>0$ such that

$$
\left|\frac{e^{-w k \ell}}{\left(e^{-w k \ell}-1\right)^{2}}-\frac{1}{\ell^{2} k^{2} w^{2}}+\frac{1}{12}\right| \leq M|k w \ell|^{2}
$$

since

$$
\lim _{z \rightarrow 0} \frac{1}{z^{2}}\left(\frac{e^{z}}{\left(e^{z}-1\right)^{2}}-\frac{1}{z^{2}}+\frac{1}{12}\right)=\frac{1}{240} .
$$

On the other hand, for $|k w \ell|>\pi$,

$$
\left|\frac{e^{-w k \ell}}{\left(e^{-w k \ell}-1\right)^{2}}\right| \leq \frac{e^{-\Re w k \ell}}{\left(e^{-\Re w k \ell}-1\right)^{2}} \leq \frac{2}{\left(1-e^{-\Re w \frac{\pi}{|w|}}\right)^{3}} .
$$

So we have the bound for $\Re w>0$

$$
\left|\frac{e^{-w k \ell}}{\left(e^{-w k \ell}-1\right)^{2}}-\frac{1}{\ell^{2} k^{2} w^{2}}+\frac{1}{12}\right| \leq \frac{2}{\left(1-e^{-\Re w \frac{\pi}{|w|}}\right)^{3}}+1
$$

The proof is now completed in the same way as Lemma 4 .

\section{Phases}

For convenience, we record a result from our paper [3].

Definition 6. 3, Definition 1] Let $\left\{L_{k}(x)\right\}$ be any sequence of complex-valued functions on a domain $D$. The set $R(m)$ is the $m$-th phase (or phase $m$ ) of $\left\{L_{k}(x)\right\}$ if (1) if $x \in R(m)$, then $\Re L_{m}(x)>\Re L_{k}(x)$ for all $k \neq m$ and (2) if $V$ is any open subset of $D$ satisfying (1), then $V \subset R(m)$. 
Theorem 7. (Parry-Boyer [3]) Let D be the punctured open unit disk and let $\left\{L_{k}(x)\right\}$ be given as in (2). Then $D$ contains exactly two nonempty phases $R(1)$ and $R(2)$ whose union is dense in $D$ and whose common boundary is the level set $\left\{x \in D: \Re L_{1}(x)=\Re L_{2}(x)\right\}$. It has exactly one real point $x^{*} \simeq-0.8250030529$ and its closure contains exactly two points $e^{ \pm i \theta^{*}}$ on the unit circle where $\theta^{*} \simeq$ $\pm 0.9517031251 \pi$. Further $R(2)$ lies in the open left half plane and $R(2) \cap \mathbb{R}=$ $\left(-1, x^{*}\right)$.

\section{Asymptotics of the Polynomials on the Phases $R(1)$ and $R(2)$}

4.1. Introduction. We will adapt the circle method which is usually used to give asymptotics for a sequence $\left\{c_{n}\right\}$ of positive numbers through their generating function $\sum_{n>0} c_{n} u^{n}$. With the coefficients $c_{n}$ replaced with the polynomials $Q_{n}(x)$, we find that the dominant contribution to their asymptotics depends on the location of $x$ in the unit disk $\mathbb{D}$. The purpose of this section is to show that the subsets of $\mathbb{D}$ where the asymptotics have the same dominant form coincide with the phases of $\left\{L_{k}(x)\right\}$. See Andrews's classic book [1, Chapter 5] for a thorough discussion of the circle method.

TheOrem 8. Let $R(1)$ and $R(2)$ be the phases of $\left\{L_{k}(x)\right\}$ given in Theorem 7 . (a) Let $x \in X \subset R(1) \backslash\left[x^{*}, 0\right]$ be a compact set, then

$$
Q_{n}(x)=\sqrt[12]{1-x} \sqrt{\frac{L_{1}(x)}{6 \pi n^{4 / 3}}} \exp \left(\frac{3}{2} n^{2 / 3} L_{1}(x)\right)\left(1+O_{X}\left(n^{-1 / 3}\right)\right) .
$$

(b) Let $x \in X \subset R(2)$ be a compact, then

$Q_{n}(x)=(-1) \sqrt[n]{1-x^{2}} \sqrt[8]{\frac{1-x}{1+x}} \sqrt{\frac{L_{2}(x)}{6 \pi n^{4 / 3}}} \exp \left(\frac{3}{2} n^{2 / 3} L_{2}(x)\right)\left(1+O_{X}\left(n^{-1 / 3}\right)\right)$.

By the Cauchy integral formula, we have an integral expression for $Q_{n}(x)$ with integration contour the circle $|u|=e^{-2 \pi \alpha}$ where

$$
\alpha=\frac{1}{2 \pi n^{1 / 3}} \Re L_{m}(x)
$$

and $m=1$ or 2 . Then we dissect the circular contour relative to a Farey sequence $F_{N}$ of order $N=\left\lfloor\delta n^{1 / 3}\right\rfloor$ as follows

$$
\begin{aligned}
Q_{n}(x) & =\frac{1}{2 \pi i} \oint_{|u|=e^{-2 \pi \alpha}} \frac{P(x, u) d u}{u^{n+1}} \\
& =\sum_{h / k \in F_{N}} \int_{h / k-1 / k\left(k+k^{\prime}\right)}^{h / k+1 / k\left(k+k^{\prime \prime}\right)} \frac{P\left(x, e^{2 \pi(-\alpha+i \psi)}\right)}{e^{2 \pi n(-\alpha+i \psi)}} d \psi \\
& =\sum_{h / k \in F_{N}} \int_{h / k-1 / k\left(k+k^{\prime}\right)}^{h / k+1 / k\left(k+k^{\prime \prime}\right)} e^{-2 \pi i n h / k} e^{2 \pi n(\alpha-i(\psi-h / k))} P\left(s, e^{-2 \pi(\alpha-i(\psi-h / k))+2 \pi i h / k)}\right) d \psi
\end{aligned}
$$

where $h^{\prime} / k^{\prime}<h / k<h^{\prime \prime} / k^{\prime \prime}$ are consecutive elements from the Farey sequence $F_{N}$ in reduced form. By convention, we will always assume that $N>m$.

We now make the change of variables $v=\psi-h / k$ and apply the factorization in Proposition 2 to get

$$
P\left(x, e^{-2 \pi(\alpha-i v)+2 \pi i h / k)}\right)=e^{2 \pi i n h / k} \omega_{h, k, n}(x) \exp \left(\frac{L_{k}(x)^{3}}{8 \pi^{2}(\alpha-i v)^{2}}+g_{h, k}(x, 2 \pi(\alpha-i v)) .\right)
$$


We introduce the integral

$I_{h, k, n}(x)=\int_{h / k-1 / k\left(k+k^{\prime}\right)}^{h / k+1 / k\left(k+k^{\prime \prime}\right)} \exp \left(\frac{L_{k}(x)^{3}}{8 \pi^{2}(\alpha-i v)^{2}}+2 \pi n(\alpha-i v)\right) e^{g_{h, k}(x, 2 \pi(\alpha-i v))} d v$.

So we may write $Q_{n}(x)$ as

$$
Q_{n}(x)=\sum_{h / k \in F_{N}} \omega_{h, k, n}(x) I_{h, k, n}(x) .
$$

Our next goal is to show that $\omega_{1, m, n}(x) I_{1, m, n}(x)$ is the dominant term in this expansion.

4.2. Major arcs. We start by making another change of variables $z=2 \pi n^{1 / 3} v$ in $I_{h, k, n}(x)$ to get

$$
\begin{aligned}
& I_{h, k, n}(x)=\frac{1}{2 \pi n^{1 / 3}} \int_{-\frac{2 \pi n^{1 / 3}}{m+m^{\prime}}}^{\frac{2 \pi n^{1 / 3}}{m+m^{\prime \prime}}} \exp [\left.n^{2 / 3}\left(\frac{L_{m}(x)^{3}}{2\left(\Re L_{m}(x)-i z\right)^{2}}+\left(\Re L_{m}(x)-i z\right)\right)\right] \\
& \times \exp \left[g_{h, k}\left(x, \frac{1}{n^{1 / 3}}\left(\Re L_{m}(x)-i z\right)\right)\right] d z .
\end{aligned}
$$

We decompose $I_{h, k, n}(x)$ into the sum $I_{h, k, n}^{\prime}(x)+I_{h, k, n}^{\prime \prime}(x)$ where

$I_{h, k, n}^{\prime}(x)=\frac{1}{2 \pi n^{1 / 3}} \int_{-\frac{2 \pi n^{1 / 3}}{m+m^{\prime}}}^{\frac{2 \pi n^{1 / 3}}{m+m^{\prime \prime}}} \exp \left[n^{2 / 3}\left(\frac{L_{m}(x)^{3}}{2\left(\Re L_{m}(x)-i z\right)^{2}}+\left(\Re L_{m}(x)-i z\right)\right)\right] d z$.

and

$$
\begin{aligned}
I_{h, k, n}^{\prime \prime}(x)=\frac{1}{2 \pi n^{1 / 3}} \int_{-\frac{2 \pi n^{1 / 3}}{m+m^{\prime}}}^{\frac{2 \pi n^{1 / 3}}{m+m^{\prime \prime}}} \exp \left[n^{2 / 3}\left(\frac{L_{m}(x)^{3}}{2\left(\Re L_{m}(x)-i z\right)^{2}}+\left(\Re L_{m}(x)-i z\right)\right)\right] \\
\times \quad\left\{\exp \left[g_{h, k}\left(x, \frac{1}{n^{1 / 3}}\left(\Re L_{m}(x)-i z\right)\right)\right]-1\right\} d z .
\end{aligned}
$$

We will obtain an asymptotic expansion of $I_{h, m, n}^{\prime}(x)$ using a saddle point expansion. To begin we need a basic inequality.

Lemma 9. Let $\alpha>0, v \in \mathbb{R}$, and $|\arg (L)| \leq \pi / 3$, then

$$
\Re\left(\frac{L^{3}}{(\alpha-i v)^{2}}\right) \leq \frac{(\Re L)^{3}}{\alpha^{2}} .
$$

If $|\arg (L)|<\pi / 3$ and $L \neq 0$, then equality is attained uniquely at $v=-\Im L$. If $|\arg (L)|=\pi / 3$ and $L \neq 0$, then equality is attained only at $v= \pm \Im L$.

Lemma 10. Let $x \in X \subset D$ be compact. For $0<\delta<\pi / \sqrt[3]{2 \zeta(3)}$, then (a) for every $x \in X, \pm \Im L_{m}(x) \in(-\pi / m \delta, \pi / m \delta) \subset\left[-\frac{2 \pi n^{1 / 3}}{m+m^{\prime}}, \frac{2 \pi n^{1 / 3}}{m+m^{\prime \prime}}\right]$.

(b) there exists a constant $K>0$ such that

$$
\begin{aligned}
I_{h, m, n}^{\prime}(x) & =\frac{1}{2 \pi n^{1 / 3}} \int_{-\frac{\pi}{m \delta}}^{\frac{\pi}{m \delta}} \exp \left[n^{2 / 3}\left(\frac{L_{m}(x)^{3}}{\left.2\left(\Re L_{m}(x)-i z\right)^{2}-i z\right)^{2}}+\left(\Re L_{m}(x)-i z\right)\right)\right] d z \\
& +O\left(\exp \left(n^{2 / 3}\left[\frac{3}{2} \Re L_{m}(x)-K\right]\right)\right) .
\end{aligned}
$$


Proof. (a) Since the Farey sequence has order $\left\lfloor\delta n^{1 / 3}\right\rfloor$, we have

$$
\frac{\pi}{m \delta} \leq \frac{2 \pi n^{1 / 3}}{m+m^{\prime}}, \quad \frac{2 \pi n^{1 / 3}}{m+m^{\prime \prime}} \leq \frac{2 \pi n^{1 / 3}}{m\left\lfloor\delta n^{1 / 3}\right\rfloor}
$$

since $h^{\prime} / m^{\prime}<h / m<h^{\prime \prime} / m^{\prime \prime}$ are consecutive terms of the Farey sequence. In particular, with $0<\delta<\pi / \sqrt[3]{2 \zeta(3)}$, we find $\left|\Im L_{m}(x)\right| \leq \frac{1}{m} \sqrt[3]{2 \zeta(3)}<\pi / m \delta$.

(b) By Lemma 9, if $|v| \geq \pi / m \delta$, there exists $K>0$ such that for all $x \in X$

$$
\Re\left(\frac{L_{m}(x)^{3}}{\left.2\left(\Re L_{m}(x)-i z\right)^{2}-i z\right)^{2}}+\left(\Re L_{m}(x)-i z\right)\right) \leq \frac{3}{2} \Re L_{m}(x)-K .
$$

We consider the estimates for the integral on the set $J=\left[-\frac{2 \pi n^{1 / 3}}{m+m^{\prime}}, \frac{2 \pi n^{1 / 3}}{m+m^{\prime \prime}}\right] \backslash$ $\left(-\frac{\pi}{m \delta}, \frac{\pi}{m \delta}\right)$ :

$$
\begin{aligned}
\left|\int_{J} \exp \left[n^{2 / 3}\left(\frac{L_{m}(x)^{3}}{2\left(\Re L_{m}(x)-i z\right)^{2}}+\left(\Re L_{m}(x)-i z\right)\right)\right] d z\right| \\
=\int_{J} \exp \left[n^{2 / 3}\left(\frac{L_{m}(x)^{3}}{2\left(\Re L_{m}(x)-i z\right)^{2}}+\left(\Re L_{m}(x)-i z\right)\right)\right] d z \\
\leq \int_{J} \exp \left[n^{2 / 3}\left(\frac{3}{2} \Re L_{m}(x)-K\right)\right] d z \\
=O\left(n^{1 / 3} \exp \left[n^{2 / 3}\left(\frac{3}{2} \Re L_{m}(x)-K\right)\right]\right) .
\end{aligned}
$$

The estimate in part (b) now follows.

Proposition 11. (a) Let $x \in X \subset \mathbb{D} \backslash\left\{x^{m} \leq 0\right\}$ be compact. Then

$$
I_{h, m, n}^{\prime}(x)=\frac{1}{\sqrt{2 \pi n^{2 / 3}}} \sqrt{\frac{L_{m}(x)}{3}} \exp \left(\frac{3}{2} n^{2 / 3} L_{m}(x)\right)\left(1+O_{X}\left(n^{-2 / 3}\right)\right) .
$$

(b) Let $x \in X \subset\left\{x \in D: x^{m} \leq 0\right\}$ be compact. Then we have

$$
\begin{aligned}
I_{h, m, n}^{\prime}(x)=\frac{1}{\sqrt{2 \pi n^{2 / 3}}}\left[\sqrt{\frac{L_{m}(x)}{3}} e^{\frac{3}{2} n^{2 / 3} L_{m}(x)}\right]\left(1+O_{X}\left(n^{-2 / 3}\right)\right) \\
\quad+\frac{1}{\sqrt{2 \pi n^{2 / 3}}}\left[\sqrt{\frac{L_{m}(x)}{3}} e^{\frac{3}{2} n^{2 / 3} L_{m}(x)}\right]^{-}\left(1+O_{X}\left(n^{-2 / 3}\right)\right) .
\end{aligned}
$$

Proof. We apply the saddle point method as given in [5, p. 10-11] and note that standard arguments will make the estimates there uniform for $x \in X$. We let $B(z)=\frac{L_{m}(x)^{3}}{2\left(\Re L_{m}(x)-i z\right)^{2}}+\left(\Re L_{m}(x)-i z\right)$.

(a) By the inequality in Lemma 9, $B(z)$ has a unique maximum on $\mathbb{R}$ at $z_{0}=$ $-\Im L_{m}(x)$ with $B\left(z_{0}\right)=\frac{3}{2 \Re L_{m}(x)}>0$. Note that uniqueness follows since, for $x \in X, \operatorname{Li}_{3}(x)<0$ if and only if $x<0$. Now the second derivative $B^{\prime \prime}(z)=$ $-3 /\left(\Re L_{m}(x)-i z\right)^{4}$ is Lipschitz continuous on $\mathbb{R}$ and $B^{\prime \prime}\left(z_{0}\right)=-3 / L_{m}(x)$. Hence, by [5. 11], we find that as $n \rightarrow \infty$

$$
\int_{-\frac{\pi}{m \delta}}^{\frac{\pi}{m \delta}} e^{n^{2 / 3} B(z)} d z=e^{n^{2 / 3} B\left(z_{0}\right)} \sqrt{\frac{\pi}{n^{2 / 3} \cdot \frac{3}{2} \frac{1}{L_{m}(x)}}}\left(1+O_{X}\left(n^{-2 / 3}\right)\right)
$$

which simplifies to the desired expression. 
(b) Let $z_{0}=\Im L_{m}(x)$. Since $x^{m}<0$, for $z \in \mathbb{R}, B(z)$ has two equal maxima at $\pm z_{0}$ with $B\left( \pm z_{0}\right)=\frac{3}{2} L_{m}(x)$. Since $\left|\arg L_{m}(x)\right|=\pi / 3$ and $x \neq 0, \Im L_{m}(x) \neq 0$. Now $B(-z)=\overline{B(z)}$ for $z \in \mathbb{R}$ since $L^{3}$ is real. We get desired the equation by applying the saddle point method as in part (a) to $\int_{0}^{\frac{\pi}{m \delta}} e^{n^{2 / 3} B(z)} d z$ and observing that $\left[\int_{0}^{\frac{\pi}{m \delta}} e^{n^{2 / 3} B(z)} d z\right]^{-}=\int_{-\frac{\pi}{m \delta}}^{0} e^{n^{2 / 3} B(z)} d z$.

Note that bounds that come from the above saddle point asymptotics must exclude the interval $\left[x^{*}, 0\right]$ from the phase $R(1)$. This explains choosing between $R(1)$ and $R(1) \backslash\left[x^{*}, 0\right]$ as a region below.

\subsection{Estimates for Non-dominant Contributions.}

4.3.1. Bounds for the Integrals $I_{h, m, n}^{\prime \prime}(x)$.

Lemma 12. Let $x \in X \subset D$ be compact with $M_{X}=\max \{|x|: x \in X\}$. Fix $\delta$ so $0<\delta<1 / \sqrt[3]{2 \zeta(3)}$ and $n \geq 1 / \delta^{3}$. Then

(a) For $k \in \mathbb{N}$ fixed, then as $n \rightarrow \infty$

$$
\left|g_{h, k}\left(x, \frac{1}{n^{1 / 3}}\left(\Re L_{m}(x)-i z\right)\right)\right|=O_{X, k}\left(\frac{1}{\delta n^{1 / 3}}\right) .
$$

(b) For $k \leq\left\lfloor\delta n^{1 / 3}\right\rfloor$, then as $n \rightarrow \infty$

$$
\left|g_{h, k}\left(x, \frac{1}{n^{1 / 3}}\left(\Re L_{m}(x)-i z\right)\right)\right| \leq \frac{2 \sqrt{5}}{1-M_{X}} \delta^{2} n^{2 / 3}+O_{X}\left(\frac{1}{\delta n^{1 / 3}}\right) .
$$

Proof. We will bound the terms in equation (7) individually. For convenience, we work with the variable $v=2 \pi n^{1 / 3} z$. Recall that $\sqrt[3]{2 \zeta(3)}<1 / \delta$ and $|v| \leq$ $\frac{1}{k N}<\frac{1}{k \delta n^{1 / 3}}$, where $N=\left\lfloor\delta n^{1 / 3}\right\rfloor$ is the order of the Farey fractions. Introduce $C_{X}=\max \left\{1 / \Re L_{m}(x): x \in X\right\}$. We start with the easy estimate:

$$
|w|=\left|\frac{\Re L_{m}(x)}{n^{1 / 3}}-i v\right| \leq \frac{1}{n^{1 / 3}} \sqrt{(2 \zeta(3))^{2 / 3}+\frac{1}{(k \delta)^{2}}} \leq \frac{\sqrt{5}}{\delta n^{1 / 3}}
$$

since $\sqrt[3]{2 \zeta(3)}$ is an upper bound for $\Re L_{m}(x), x \in X$.

Next by using that $1 /\left(1-e^{-t}\right)<1+1 / t$ for $t>0$, we can show that

$$
\frac{1}{1-e^{-\frac{\pi \Re w}{k|\Im w|}}} \leq 1+\frac{\sqrt{5} C_{X}}{\pi \delta}, \quad \frac{1}{1-e^{-\frac{\pi \Re w}{|w|}}} \leq 1+\frac{\sqrt{5} C_{X}}{\pi \delta}
$$

Using $e^{-t} \leq 27 /(e t)^{3}$ for $t>0$, we obtain

$$
|x|^{\frac{\pi}{k \mid \Im w} \mid} \leq \frac{-27}{\left(2 \pi e n^{1 / 3} \delta \ln M_{X}\right)^{3}}, \quad|x|^{\frac{\pi}{w \mid}} \leq \frac{-27 \sqrt{125}}{\left(\pi e \delta n^{1 / 3} \ln M_{X}\right)^{3}} .
$$

Hence, $\left|g_{h, k}\left(x, \frac{\Re L_{m}(x)}{n^{1 / 3}}-i v\right)\right|$ is bounded above by

$$
\begin{aligned}
& \frac{2 \sqrt{5}}{\delta n^{1 / 3}\left(1-M_{X}\right)}\left[k^{3}+\frac{-27}{\left(2 \pi e \delta n^{1 / 3} \ln M_{X}\right)^{3}} \cdot\left(1+\frac{2 C_{X}}{\pi \delta}\right)\right] \\
& \quad+\frac{1}{\left(1-M_{X}\right)^{2}}\left[\frac{5 M k}{\delta^{2} n^{2 / 3}}+\left(2 \cdot\left(1+\frac{\sqrt{5} C_{X}}{\pi \delta}\right)^{3}+1\right) \cdot \frac{-27}{\left(\pi e \delta n^{1 / 3} \ln M_{X}\right)^{3}}\right] .
\end{aligned}
$$


We rewrite this upper bound as a polynomial in $k$ with constants $A$ and $B$ independent of $\delta, k$, and $n$ :

$$
\frac{2 \sqrt{5}}{\delta n^{1 / 3}\left(1-M_{X}\right)} k^{3}+\frac{1}{\left(1-M_{X}\right)^{2}} \frac{5 M}{\delta^{2} n^{2 / 3}} k+A \frac{1}{\delta^{5} n^{4 / 3}}+B \frac{1}{\delta^{6} n} .
$$

With $k$ fixed, we obtain part (a) since the bound in (12) is $O_{X}\left(1 /\left[\delta n^{1 / 3}\right]\right)$; while if we replace $k$ with $\delta n^{1 / 3}$, we get the bound in part (b).

Lemma 13. Let $x \in X$ where $X$ is a compact subset of either $R(1) \backslash\left[x^{*}, 0\right]$ or $R(2)$. Then $I_{h, m, n}^{\prime \prime}(x)=O_{X, \delta}\left(I_{h, m, n}^{\prime}(x) / n^{1 / 3}\right), m=1,2$.

Proof. By the definition of $I_{h, m, n}^{\prime \prime}(x)$ given in subsection 4.2, it is enough to observe that there exists a positive constant $K_{X, m, \delta}$ such that

$$
\left|e^{g_{h, m}\left(x, \frac{1}{n^{1 / 3}}\left(\Re L_{m}(x)-i z\right)\right)}\right| \leq \frac{K_{X, m, \delta}}{n^{1 / 3}}
$$

by the above lemma.

\subsubsection{Bounds for Minor Arcs.}

Lemma 14. Let $x \in X$ where $X$ is a compact subset of unit disk. Then

$$
\left|\omega_{h, k, n}(x)\right| \leq 2^{1 / 12} \exp \left(\frac{k^{2}}{16 h^{2}} L i_{3}\left(M_{X}\right)\right) \leq 2^{1 / 12} \exp \left(\frac{k^{2}}{16}\left(\zeta(3)-\ln \left(1-M_{X}\right)\right)\right)
$$

where $M_{X}=\max \{|x|: x \in X\}$ and $\omega_{h, k, n}(x)$ is given in (5).

Proof. We bound each component separately in the definition of $\omega_{h, k, n}(x)$. We start with the first factor

$$
\left|\exp \left(\frac{1}{12 k} \ln \left(1-x^{k}\right)\right)\right|=\exp \left(\frac{1}{12 k} \Re \ln \left(1-x^{k}\right)\right) \leq 2^{1 / 12 k} .
$$

Next we obtain an intermediate bound

$$
\left|\exp \left(-\frac{1}{4} \sum_{\ell \nmid k} \frac{x^{\ell}}{\ell} \csc ^{2}(\pi h \ell / k)\right)\right| \leq \exp \left(\frac{1}{4} \sum_{\ell \nmid k} \frac{|x|^{\ell}}{\ell} \csc ^{2}(\pi h \ell / k)\right) .
$$

Break up the above series into two sums with the sets of indices

$$
J_{1}=\{\ell: \ell \nmid k, h \ell / k \bmod 1<1 / 2\}, \quad J_{2}=\{\ell \nmid k, 1 / 2 \geq h \ell / k \bmod 1\} .
$$

and use the elementary estimates $\sin \theta \geq 2 \theta / \pi$, for $0 \leq \theta \leq \pi / 2$, and $\geq 2(1-\theta / \pi)$ for $\pi / 2 \leq \theta \leq \pi$ to obtain the two bounds below to complete the proof:

$$
\begin{aligned}
& \exp \left(\frac{1}{4} \sum_{\ell \in J_{1}} \frac{|x|^{\ell}}{\ell} \csc ^{2}(\pi h \ell / k)\right) \leq \exp \left(\frac{k^{2}}{16 h^{2}} L i_{3}\left(M_{X}\right)\right), \\
& \exp \left(\frac{1}{4} \sum_{\ell \in J_{2}} \frac{|x|^{\ell}}{\ell} \csc ^{2}(\pi h \ell / k)\right) \leq \exp \left(-\frac{k^{2}}{16} \ln \left(1-M_{X}\right)\right) .
\end{aligned}
$$


Lemma 15. Let $x \in X$ where $X$ is a compact subset of $R(m), m=1,2$. There exists a positive constant $a_{X}$ such that

$$
\Re\left[\frac{L_{k}(x)^{3}}{8 \pi^{2}(\alpha-i v)^{2}}+2 \pi n(\alpha-i v)\right] \leq n^{2 / 3}\left(\frac{3}{2} \Re L_{m}(x)+a_{X}\right), \quad k \neq m, x \in X,
$$

where $\alpha$ is given in equation (9).

Proof. In subsection 4.2 we saw that

$$
\begin{aligned}
\frac{1}{n^{2 / 3}} \Re\left[\frac{L_{k}(x)^{3}}{8 \pi^{2}(\alpha-i v)^{2}}\right. & +2 \pi n(\alpha-i v)] \\
= & \Re\left[\frac{L_{k}(x)^{3}}{2\left(\Re L_{m}(x)-2 \pi i n^{1 / 3} v\right)^{2}}+\left(\Re L_{m}(x)-2 \pi i n^{1 / 3} v\right)\right] .
\end{aligned}
$$

By Lemma 9, we have the strict bound for $x \in X$ and $k \neq m$

$$
\begin{aligned}
\Re\left[\frac{L_{k}(x)^{3}}{2\left(\Re L_{m}(x)-2 \pi i n^{1 / 3} v\right)^{2}}+\left(\Re L_{m}(x)-2 \pi i n^{1 / 3} v\right)\right] \\
\leq \frac{\left(\Re L_{k}(x)\right)^{3}}{2\left(\Re L_{m}(x)\right)^{2}}+\Re L_{m}(x)<\frac{3}{2} \Re L_{m}(x) .
\end{aligned}
$$

Hence the difference, for $k \neq m$, has a positive minimum $a_{X, k}$ on $X$ :

$\frac{3}{2} \Re L_{m}(x)-\Re\left[\frac{L_{k}(x)^{3}}{2\left(\Re L_{m}(x)-2 \pi i n^{1 / 3} v\right)^{2}}+\left(\Re L_{m}(x)-2 \pi i n^{1 / 3} v\right)\right] \geq a_{X, k}>0, x \in X$,

by compactness. Consider $a_{X, k}$ as a sequence, then it converges to the minimum of $\frac{3}{2} \Re L_{m}(x)$ on $X$ which is positive. Hence, $\inf \left\{a_{X, k}: k \neq m\right\}$ is attained for some index, say $k_{0} \neq m$. In particular, we have

$$
a_{X, k} \geq a_{X, k_{0}}>0, \quad k \neq m .
$$

For simplicity, we write $a_{X}$ for $a_{X, k_{0}}$. The inequality in the lemma follows.

\subsection{Conclusion of proof of Theorem 8 .}

Lemma 16. Let $x \in X$ be a compact subset of $R(m), m=1,2$. Then there exists $\delta_{0}>0$ and $\eta>0$ such that

$$
\left|\sum_{h / k \in F_{N}, k \neq m} \omega_{h, k, n}(x) I_{h, k, n}(x)\right| \leq 2^{1 / 12} \exp \left(\left(\frac{3}{2} \Re L_{m}(x)-\eta\right) n^{2 / 3}+O_{X, \delta_{0}}(1)\right)
$$

Proof. We need to estimate $I_{h, k, n}(x)$ where $k \neq m$ :

$$
I_{h, k, n}(x)=\omega_{h, k, n}(x) \int_{-\frac{1}{k\left(k+k^{\prime}\right)}}^{\frac{1}{k\left(k+k^{\prime \prime}\right)}} \exp \left(\frac{L_{k}(x)^{3}}{8 \pi^{2}(\alpha-i v)^{2}}+2 \pi n(\alpha-i v)\right) \exp \left(g_{h, k}(x, 2 \pi(\alpha-i v))\right) d v \text {. }
$$

We begin with the bounds

$$
\begin{aligned}
& \left|\int_{-\frac{1}{k\left(k+k^{\prime}\right)}}^{\frac{1}{k\left(k+k^{\prime \prime}\right)}} \exp \left(\frac{L_{k}(x)^{3}}{8 \pi^{2}(\alpha-i v)^{2}}+2 \pi n(\alpha-i v)\right) \exp \left(g_{h, k}(x, 2 \pi(\alpha-i v))\right) d v\right| \\
& \quad \leq \int_{-\frac{1}{k\left(k+k^{\prime \prime}\right)}}^{\frac{1}{k\left(k+k^{\prime}\right)}}\left|\exp \left(\frac{L_{k}(x)^{3}}{8 \pi^{2}(\alpha-i v)^{2}}+2 \pi n(\alpha-i v)\right)\right|\left|\exp \left(g_{h, k}(x, 2 \pi(\alpha-i v))\right)\right| d v \\
& \quad \leq \int_{-\frac{1}{k\left(k+k^{\prime \prime}\right)}}^{\frac{1}{k\left(k+k^{\prime}\right)}} \exp \left(-a_{X} n^{2 / 3}+\frac{3}{2} n^{2 / 3} \Re L_{m}(x)\right)\left|\exp \left(\frac{2 \sqrt{2}}{1-M_{X}} \delta^{2} n^{2 / 3}+o_{X, \delta}(1)\right)\right| d v
\end{aligned}
$$


where we used Lemma 12. Hence a full bound for the sum over $h / k \in F_{N}, k \neq m$ is

$$
\begin{aligned}
& 2^{1 / 12} \exp \left(-a_{X, \delta} n^{2 / 3}+\frac{3}{2} n^{2 / 3} \Re L_{m}(x)+\frac{2 \sqrt{2}}{1-M_{X}} \delta^{2} n^{2 / 3}\right. \\
& \left.\quad+\delta^{2} n^{2 / 3} \frac{\zeta(3)}{16}-\delta^{2} n^{2 / 3} \frac{\ln \left(1-M_{X}\right)}{16}+O_{X, \delta}(1)\right)\left(\frac{1}{k\left(k+k^{\prime}\right)}+\frac{1}{k\left(k+k^{\prime \prime}\right)}\right) .
\end{aligned}
$$

where the last factor is the length of the interval of integration. Note that this bound holds for any $h / k \in F_{N}$ with $k \neq m$.

Let $\delta_{0}>0$ be chosen so that

$$
a_{X}-\frac{2 \sqrt{2}}{1-M_{X}} \delta_{0}-\frac{\zeta(3)}{16} \delta_{0}+\frac{\ln \left(1-M_{X}\right)}{16} \delta_{0}>0 .
$$

Set $\eta=a_{X}-\frac{2 \sqrt{2}}{1-M_{X}} \delta_{0}-\frac{\zeta(3)}{16} \delta_{0}+\frac{\ln \left(1-M_{X}\right)}{16} \delta_{0}>0$ so we now write new full bound as

$$
2^{1 / 12} \exp \left(\left(\frac{3}{2} \Re L_{m}(x)-\eta\right) n^{2 / 3}+O_{X, \delta_{0}}(1)\right) .
$$

The bound for the contributions over all the minor arcs is

$$
\left|\sum_{h / k \in F_{N}, k \neq m} \omega_{h, k, n}(x) I_{h, k, n}(x)\right| \leq 2^{1 / 12} \exp \left(\left(\frac{3}{2} \Re L_{m}(x)-\eta\right) n^{2 / 3}+O_{X, \delta_{0}}(1)\right)
$$

since the sum of the lengths of all the minor arcs is less than 1 . The proof is complete.

Proof. We now complete the proof of Theorem 8 . Choose the order $N$ of the Farey fractions to be $\left\lfloor\delta_{0} n^{1 / 3}\right\rfloor$ where $\delta_{0}$ is given in Lemma 16. By equation (11), we write

$$
Q_{n}(x)=\omega_{1, m, n}(x) I_{1, m, n}(x)+\sum_{h / k \in F_{N}, k \neq m} \omega_{h, k, n}(x) I_{h, k, n}(x)
$$

By Proposition 11 and Lemma 13, we see that

$$
\omega_{1, m, n}(x) I_{1, m, n}(x)=\omega_{1, m, n}(x) \sqrt{\frac{L_{m}(x)}{6 \pi n^{4 / 3}}} \exp \left(\frac{3}{2} n^{2 / 3} L_{m}(x)\right)\left(1+O_{X}\left(n^{-1 / 3}\right)\right)
$$

which dominates the contribution of the sum over $h / k \in F_{N}, k \neq m$ by Lemma 16 . Finally, we observe that

$$
\omega_{1,1, n}(x)=\sqrt[12]{1-x}, \quad \omega_{1,2, n}(x)=(-1)^{n} \sqrt[24]{1-x^{2}} \sqrt[8]{\frac{1-x}{1+x}}
$$

to complete the proof.

\section{Asymptotics on the Boundaries of the Phases $R(1)$ and $R(2)$}

Let $x \in X \subset\left\{x: \Re L_{1}(x)=\Re L_{2}(x), x \neq x^{*}\right\}$ be compact. Then the choice of $\alpha$ in equation (9) must satisfy

$$
\alpha=\frac{1}{2 \pi n^{1 / 3}} \Re L_{1}(x)=\frac{1}{2 \pi n^{1 / 3}} \Re L_{2}(x)
$$

so both terms $\omega_{1,1, n} I_{1,1, n}(x)$ and $\omega_{1,2, n}(x) I_{1,2, n}(x)$ will contribute to the dominant asymptotics of $Q_{n}(x)$. A similar modification must be used for the asymptotics on the compact subsets of $\left(x^{*}, 0\right)$. In this case, the two terms are $\omega_{1,1, n} I_{1,1, n}(x)$ and its 
complex conjugate which combine to give an oscillatory term. Further simplification occurs because the argument of $L_{1}(x)$ is constant on $\left(x^{*}, 0\right)$. We record the results:

THEOREM 17. Let $x^{*}$ be the negative real number given in Theorem 7 . (a) Let $x \in X \subset\left(x^{*}, 0\right)$ be compact. Then

$$
\begin{aligned}
Q_{n}(x)=2^{7 / 6} \sqrt[12]{1-x} & \frac{1}{\sqrt{6 \pi n^{4 / 3}}}\left|L i_{3}(x)\right|^{1 / 6} \exp \left(\frac{3}{4} \sqrt[3]{2} n^{2 / 3}\left|L i_{3}(x)\right|^{1 / 3}\right) \\
& \times\left(\cos \left(\frac{3 \sqrt{3}}{4} \sqrt[3]{2} n^{2 / 3}\left|L i_{3}(x)\right|^{1 / 3}+\frac{\pi}{6}\right)+O_{X}\left(n^{-1 / 3}\right)\right) .
\end{aligned}
$$

(b) Let $x \in X \subset\left\{x: \Re L_{1}(x)=\Re L_{2}(x), x \neq x^{*}\right\}$ be compact. Then

$$
\begin{aligned}
Q_{n}(x)=\sqrt[12]{1-x} \sqrt{\frac{L_{1}(x)}{6 \pi n^{4 / 3}}} \exp \left(\frac{3}{2} n^{2 / 3} L_{1}(x)\right)\left(1+O_{X}\left(n^{-1 / 3}\right)\right) \\
+(-1) \sqrt[n]{\frac{24}{1-x}} \sqrt[8]{\frac{1-x}{1+x}} \sqrt{\frac{L_{2}(x)}{6 \pi n^{4 / 3}}} \exp \left(\frac{3}{2} n^{2 / 3} L_{2}(x)\right)\left(1+O_{X}\left(n^{-1 / 3}\right)\right) .
\end{aligned}
$$

Note that in part (a) above, we are abusing notation since this relation holds only if $\Re\left(\omega_{1,1, n}(x) I_{1,1, n}(x)\right)$ is nonzero. On the other hand, if $\Re\left(\omega_{1,1, n}(x) I_{1,1, n}(x)\right)=$ 0 holds, $Q_{n}(x)$ reduces to $O\left(\omega_{1,1, n}(x) I_{1,1, n}(x) n^{-1 / 3}\right)$.

Finally, we remark that the asymptotics of $Q_{n}(x)$ over $(-1,1)$ has three separate regimes: $\left(-1, x^{*}\right),\left(x^{*}, 0\right)$, and $(0,1)$. On $\left(-1, x^{*}\right)$ and $(0,1)$, the polynomials have exponential growth while on $\left(x^{*}, 0\right)$ the polynomials are oscillating. In [4], this asymptotic was found to give good approximations to the zeros of $Q_{n}(x)$ in that interval. The partition polynomials built from the usual partition numbers and studied in [2] do not have this oscillatory behavior.

\section{References}

[1] George E. Andrews, "The Theory of Partitions," Reading, MA: Addison-Wesley Press, 1976.

[2] Robert P. Boyer and William M. Y. Goh, Partition polynomials: asymptotics and zeros, Contemporary Mathematics, Volume 457 (2008), 99-111.

[3] Robert P. Boyer and Daniel T. Parry, Phase calculations for plane partition polynomials, accepted for publication, Rocky Mountain Journal of Mathematics.

[4] Robert P Boyer and Daniel T. Parry, On the Zeros of Plane Partition Polynomials, The Electronic Journal of Combinatorics, Volume 18 (2) (2012) \#30 (26 pages)

[5] Mark A. Pinsky, "Introduction to Fourier Analysis," American Mathematical Society, Providence, RI, 2002.

[6] Richard P. Stanley, The conjugate trace and trace of a plane partition, J. Comb. Theory, Ser. A 14 (1973) 53-65.

[7] Richard P. Stanley, http://www-math.mit.edu/ rstan/zeros

[8] E. Maitland Wright, Asymptotic Partition Formulae: (I) Plane Partitions, Quart. J. Math. Oxford Ser. (2) 2 (1931) 177-189.

Department of Mathematics, Drexel University USA

E-mail address: rboyer@drexel.edu

Department of Mathematics, Drexel University USA

E-mail address: dtp23@drexel.edu 